\title{
NOVEDADES EN LOS GÉNEROS CALENIA Y TRICHARIA (GOMPHILLACEAE, ASCOMYCETES FOLIÍCOLAS LIQUENIZADOS) DE ARGENTINA Y PARAGUAY ORIENTAL
}

\author{
por LIDIA I. FERRARO
}

\section{Summary}

\begin{abstract}
In the present paper the author updates the taxonomic and floristic knowledge of foliicolous lichenized fungi, Calenia and Tricharia (Gomphillaceae), in Argentina and Paraguay. The following taxa are reported for the first time: Calenia depressa, C. phyllogena, and Tricharia purulhensis. The new taxon Tricharia urceolata var. paraguayensis is described.
\end{abstract}

Key words: Foliicolous-lichens, Calenia, Tricharia, Gomphillaceae.

Palabras claves: Líquenes foliícolas, Calenia, Tricharia, Gomphillaceae.

\section{Introducción}

La familia Gomphillaceae W. Watson ex Hafellner emend. Vezda \& Poelt está constituida por un grupo de entidades delimitadas por las siguientes características: apotecios biatorinos, paráfisis delgadas, muy ramificadas y anastomosadas, ascos fisitunicados, J negativo, esporas hialinas, desde transversalmente septadas hasta muriformes y conidióforos muy especializados denominados hifóforos.

Los hifóforos son órganos de propagación asexual, productores de conidios en estructuras muy características. Su función fue reconocida por Vezda (1973) quien también los describió. Otros autores (Cavalcante et al., 1972 y Santesson, 1952) los interpretaron como géneros de hongos parasimbiónticos y recibieron también diferentes nombres, por ejemplo fueron llamados "esporodoquios". Sérusiaux et al. (1986) describen e ilustran el desarrollo

\footnotetext{
${ }^{1}$ Instituto de Botánica del Nordeste, Casilla de Correo 209, 3400 Corrientes, Argentina.

Este trabajo es parte de la tesis doctoral que la autora está realizando en la Facultad de Ciencias Exactas, Naturales y Agrimensura de la Universidad Nacional del Nordeste, Corrientes, Argentina.
}

de los hifóforos en especies de Gyalectidium de Argentina. Vezda (1979) los clasifica, teniendo en cuenta la morfología que presentan, en dos grandes grupos: zigomorfos (Fig. 1, A-D) y radiados (Fig. 1, E-F). Entre los primeros se encuentran hifóforos pedunculados, setiformes, cerdosos o como escobilla, en forma de mano o cuchara, baciliformes y escamosos con dos prolongaciones en los extremos; éste es el tipo más frecuente. Mientras que los hifóforos radiados, fueron encontrados solamente en una especie del género Gyalideopsis Vezda, que presentan un pedúnculo central con una estructura apical radiada en la cara inferior que sostiene a los conidios. En una contribución sobre el género Gyalectidium Ferraro, Lücking \& Sérusiaux (en preparación) agregan a los tipos indicados una nueva forma de hifóforo zigomorfo, encontrado en una especie de $\mathrm{Pa}-$ raguay. Se trata de una escama angosta y muy larga, apretada sobre el talo que se eleva al humedecerse.

Los hifóforos de los géneros Calenia y Tricharia, tratados en este trabajo, son zigomorfos. Calenia presenta hifóforos cerdosos (Fig. 1, C-D), blancos o castaños y pueden o no estar presentes en los talos. Por su parte Tricharia se caracteriza por sus hifóforos de 
forma y longitud variables, desde pedunculados hasta cerdosos o como escobilla.

Otras características de los géneros de esta familia son los apotecios con desarrollo hemiangiocárpico y los ascos anelíferos (son unitunicados con tolo que forma un anillo en el lumen).

\section{Antecedentes}

La familia Gomphillnceae fue establecida por Watson (1929) como monotípica; más tarde fue ampliada por Vezda (1979) y luego por Vezda \& Poelt (1987).

La mayoría de los géneros que comprende actualmente estuvieron durante mucho tiempo incluidos en Asterothyriaceae. La familia, integrada originariamente por Asterothyrium,
Psorotheciopsis, Calenia, Gyalectidium y Tricharia, se la trata como tal en la monografía de Santesson (1952).

Más tarde, Vezda (1979) y Vezda \& Poelt (1987) excluyen los géneros Calenia Müll. Arg. emend. R. Sant., Gyalectidium Müll. Arg., Echinoplaca (Müll. Arg.) R. Sant. y Tricharia Fée emend. R. Sant., manteniendo en ella solamente a Asterothyrium y Psorotheciopsis. Actualmente, los restantes géneros quedan formalmente en Gomphillaceae. A ellos se suman Aulaxina Müll. Arg., Caleniopsis Vezda \& Poelt, Actinoplaca (Müll. Arg.) R. Sant. y Gyalideopsis Vezda.

El género tipo de la familia, Gomphillus Nyl., comprende de acuerdo a Kalb \& Vezda (1988), tres especies, G. ophiosporus Kalb \& Vezda, G. americanus Essl. y G. calicioides (Del. ex Duby) Nyl.
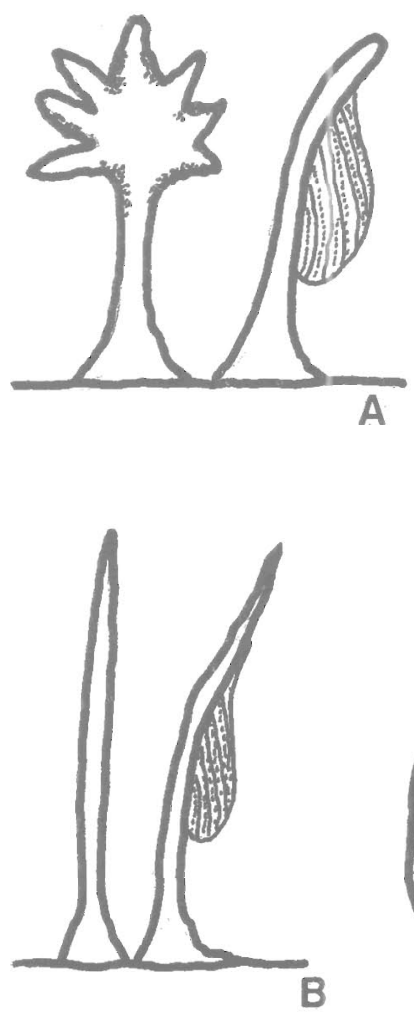
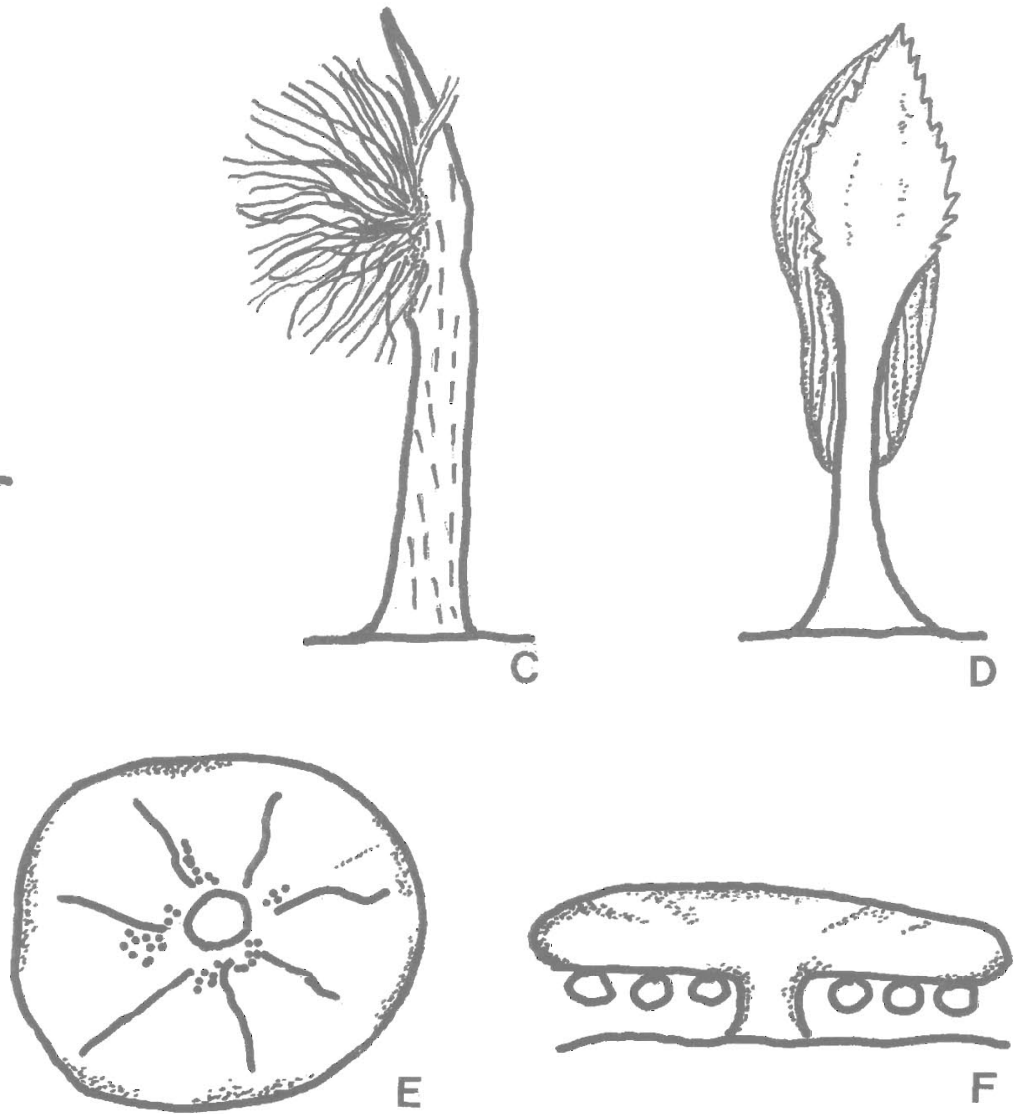

Fig. 1. A-D, hifóforos zigomorfos, cerdosos o como escobilla, vistos de frente y de costado. E-F, hifóforos radiados vistos desde arriba y de costado. 
Considerando los géneros Bullatina Vezda \& Poelt, Paratricharia Lücking e Hippocrepidea Sérusiaux, recientemente creados, la familia Gomphillaceae comprende 12 géneros con unas 135 especies distribuidas en regiones tropicales y neotropicales del mundo. En resumen, 8 géneros de Gomphillaceae y un total de 30 especies han sido estudiadas hasta el momento en Argentina y Paraguay.

\section{Material y métodos}

Los ejemplares estudiados fueron encontrados sobre hojas coleccionadas hasta una altura de $2 \mathrm{~m}$ en árboles del interior y del borde de la selva. No se realizaron colecciones en partes más altas de la copa. Algunas hojas de plantas deciduas fueron recogidas del piso de la selva y fueron analizadas, pero no se registraron ejemplares de líquenes en buen estado.

Todo el material se encuentra en el herbario CTES, duplicados fueron enviados a los herbarios privados de R. Lücking (Bayreuth, Alemania) y A. Vezda (Brno, República Checa).

Para determinar el material se hicieron cortes del talo a mano alzada, las esporas se estudiaron en cortes de apotecios y en aplastados de partes de los mismos, las medidas se tomaron midiendo 50 esporas, en los casos que fue posible. Los hifóforos fueron medidos e ilustrados con cámara clara de una lupa Wild M 5.

Es importante destacar las colecciones realizadas en las provincias del NW de Argentina, ya que los datos sobre ejemplares foliícolas de esas zonas son bastante escasos.

\section{Resultados}

Calenia Müll. Arg. emend. R. Sant.

Existen unas 20 especies de Calenia en todo el mundo, hasta el momento se han coleccionado 3 de ellas en Argentina y Paraguay Oriental.

Calenia monospora Vezda ya había sido mencionada para Corrientes, Argentina, en un catálogo (Ferraro, 1997), por lo que en esta contribución se agrega una breve descripción de la especie.

Las dos especies restantes constituyen los primeros registros para el área estudiada.

\section{Calenia depressa Müll. Arg.}

Fig. 2, A

Müller Argoviensis J., Licheni epiphylli Novi: 4. 1890. Santesson, R., Symb. Bot. Ups. 12(1): 340. 1952.

Talo verdoso-blanquecino, $1-1,5(2,5) \mathrm{cm}$, verrucoso con un hipotalo transparente de ancho variable, más o menos 0,5-1 mm, verrugas del talo pequeñas, más o menos confluentes, conteniendo grupos o racimos de cristales incoloros. Glabro o con pelos rígidos, estériles, aguzados en el extremo, claros, dispersos, escasos, hasta $1 \mathrm{~mm}$ long.

Apotecios 0,5-1,5 mm diám., circulares, solitarios o agregados, deformados, cuando están agrupados forman "superficies himeniales" de 3-5 mm. Disco primero hundido, luego prominente, castaño-grisáceo a gris-oscuro, a veces con un tinte rosado-oscuro. Los apotecios adultos con partes del disco prominentes, elevadas sobre todo en la región central y más planas hacia el borde, éste generalmente también elevado y de igual color que el talo o un poco más claro. Excípulo prosoplectenquimático, incoloro, más o menos $5 \mu \mathrm{m}$ de espesor.

Esporas con 5-7 (-8) septos transversales y a veces con uno solo longitudinal, oblongas, cilíndricas o fusiformes, constrictas en los septos, $24 \times 7-12 \mu \mathrm{m}$.

Material estudiado: ARGENTINA. Misiones, dpto. Iguazú, Parque Nacional Iguazú, sendero Macuco, Ferraro et al. 5015 (CTES); ídem, sendero Yacaratia, sobre hojas de Sorocea bonplandii (Moraceae), 8-VIII-1995; dpto. Gral. Belgrano, Colonia Andresito, Chacra 30, forófito indeterminado, 1-III-1996, Vanni et al. 3674 (CTES); dpto. Eldorado, Eldorado, sobre hojas de Bignoniaceae, 5-I-1999, Chatellenaz y Rios 23 (CTES); dpto. Guaraní, Predio Guaraní, $26^{\circ} 54^{\prime}-59^{\prime} \mathrm{S}, 54^{\circ} 12^{\prime}-18^{\prime} \mathrm{W}$, sendero a la Aldea Aborigen, en selva, sobre hojas de Sorocea, 29-IV-99, Ferraro et al. 6272 (CTES).

\section{Calenia monospora Vezda}

Fig. 2, B

Vezda, A., Folia Geobot. Phytotax. (Praha) 14: 56-57. 1979.

Talo liso, verdoso-blanquecino con células algales globosas, 5-10 mm diám., disperso hasta 

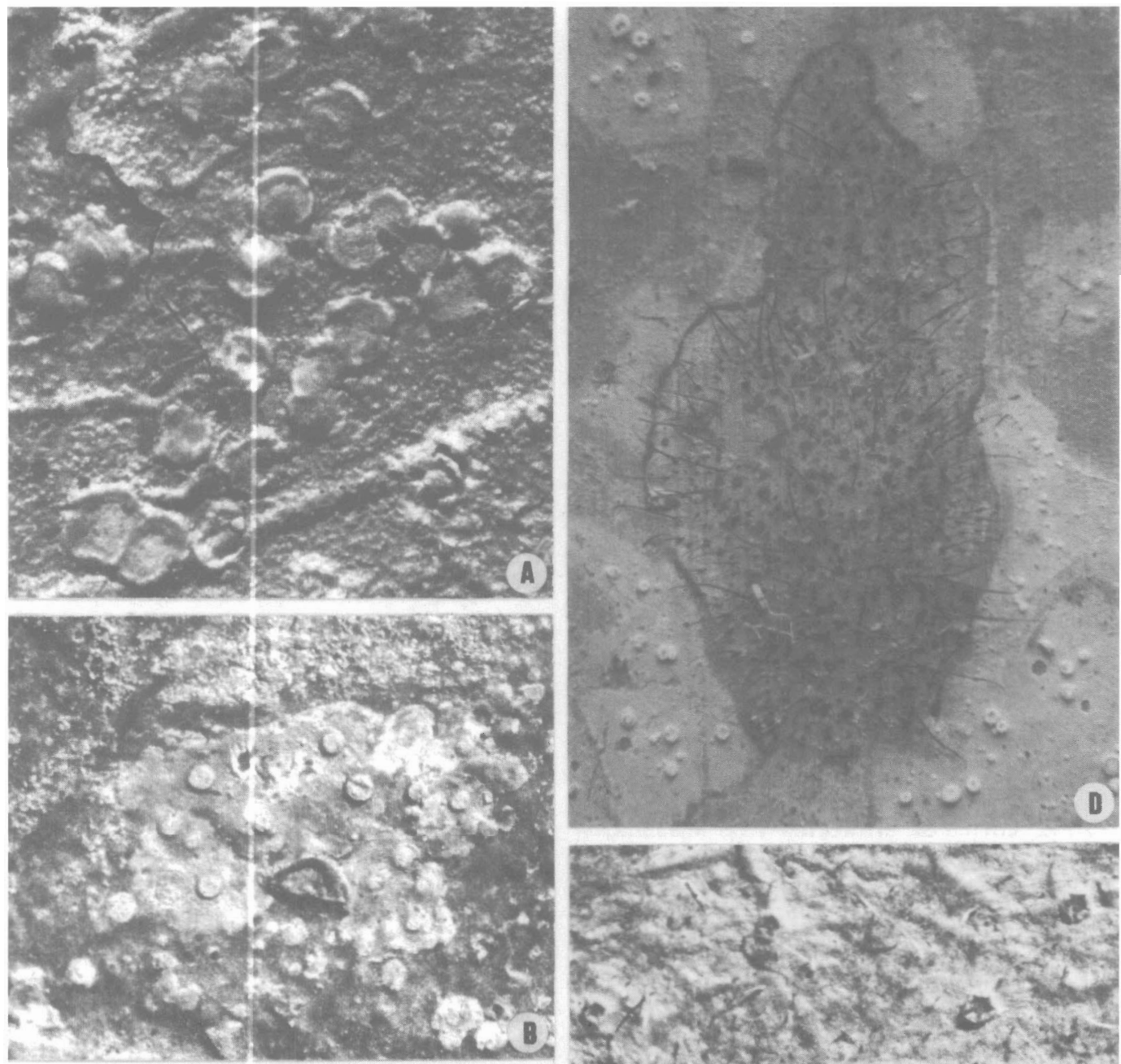

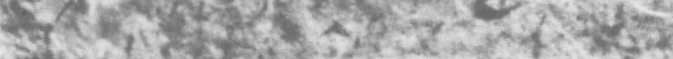
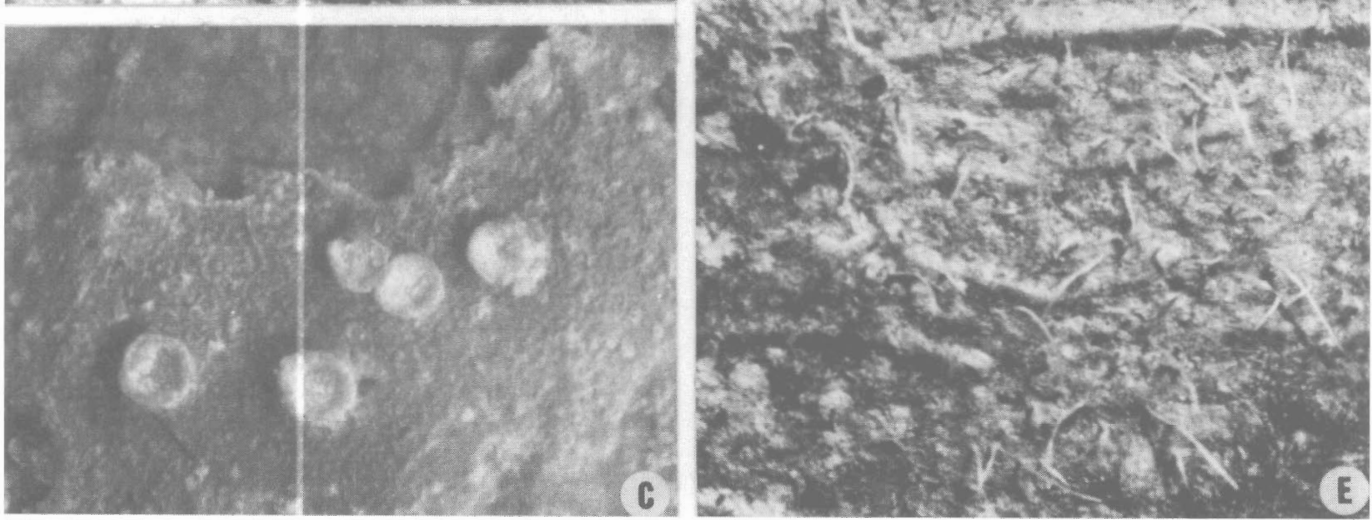

Fig. 2. A, Calenia depressa (Ferraro 5015a,CTES). B,C.monospora, apoteciosy talo sin setas (Vanni3658, CTES). C, C. phyllogena (Maruñak 887, CTES). D, Tricharia urceolata var. paraguayensis (Ferraro 3668, CTFS). E, T. purulhensis (Ferraro 5755, CTES). 
confluientes y más o menós circular. Con o sin setas o pelos, cuando presentes las setas blancas, estériles, quebradizas, aguzadas, hasta 1,5 mm long.

Apotecios 0,3-1 mm diám., circulares, cuando jóvenes más o menos inmersos en el talo y elevados en la madurez, planos, con el margen inconspicuo y de igual color que el talo y el disco también blanco-amarillento hasta algo verde-amarillento, cubierto de una fina pruina blanquecina.

Excípulo delgado, hialino. Hipotecio hialino. Ascos anchamente oblongos, uniesporados.

Esporas elipsoides, muriformes, 45-60 x 20$25 \mu \mathrm{m}$.

C. monospora es muy similar externamente a Gyalectidium filicinum Müll. Arg., sobre todo cuando no presenta los pelos o setas blancas.

Los ejemplares de Misiones se caracterizan por no presentar setas, éstas se observan en colecciones de Paraguay. El talo es liso, ecorticado y opaco, los apotecios, generalmente numerosos son del mismo color que el talo o de un característico color verde-amarillento claro, siempre circulares, no se deforman en la madurez, ni se vuelven prominentes o "robustos" como ocurre con los apotecios de G. filicinum.

El ejemplar Ferraro \& Ahti 4576 (CTES, hb. Vezda) muestra hifóforos setiformes de color castaño-rojizo pero no se ven apotecios. En este ejemplar, los talos de C. monospora se encuentran junto a otros de G. filicinum, ambos parasitados por un ascomycete aún no identificado, que presenta esporas hialinas, pequeñas, muriformes y ascos claviformes.

En el herbario CTES existen colecciones de esta especie procedentes de localidades de Brasil, Rio Grande do Sul, Iraí, sobre hojas de Pilocarpus (Rutaceae) y sobre hojas de Seguieria aculeata (Phytolaccaceae).

Material estudiado: ARGENTINA. Corrientes, dpto. Santo Tomé, Estancia Timbó, interior de monte, 5-XI-96, Maruñak et al. 953 (CTES). Formosa, dpto. Capital, Estancia Guaycolec, 22 km N de Formosa, ruta 11, en borde de selva marginal del riacho Pilagá, sobre hojas de Pilocarpus (Rutaceae), 15-II-95, Ferraro et al. 4606 (CTES). Misiones, dpto. General Belgrano, Colonia Andresito, Chacra 30, 1-III-96, Vanni et al.
3658 (CTES); dpto. Guaraní, Predio Guaraní, 26054'$59^{\prime} \mathrm{S}, 54^{\circ} 12^{\prime}-18^{\prime} \mathrm{W}$, sendero CIFOR, en interior de selva, sobre hojas de Serjania (Säpindaceae), 27-IV99, Ferraro, Tressens, Keller, Maruñak y Revilla 6121 (CTES) y sobre hojas de Pilocarpus, Ferraro et al. 6044 ${ }^{\mathrm{a}}$ (CTES); ídem, sendero a la Aldea Aborigen, cerca de la casa del guardaparque, en selva, 29-IV-99, Ferraro, Tressens, Keller, Maruñak y Revilla 6224 (CTES). Jujuy, dpto. Gral. San Martín, Parque Nacional Calilegua, sendero al camping Águas Negras, en selva, Distrito Yungas, 17-IV-98, Ferraro, López, Popoff y Vanni 5868 (CTES). PARAGUAY. Itapúa, Capitán Miranda, El Tirol, 14 km N de Encarnación, ca. $150 \mathrm{~m}$, sobre hojas de Capparis (Capparidaceae), en selva vecina al Hotel Tirol, 14-VIII-93, Ferraro y Ahti 4572 (CTES, hb. Lücking); ídem, Ferraro y Ahti 4576 (CTES, hb. Lücking) y 4589a (CTES). Canendiyú, $22 \mathrm{~km}$ de Colonia Esperanza, Reserva Biológica Itābó, $2435^{\prime} \mathrm{S}, 5448^{\prime} \mathrm{W}$, sobre frondes de helechos, 15-X-96, Schinini et al. 31569 y 31581 (CTES, hb. Lücking); ídem, sobre Ilex paraguayensis (Aquifoliaceae), 11-II-1997, Dematteis y Caballero Mármori 575 (CTES).

Calenia phyllogena (Míll. Arg.) R. Sant. Fig. 2, C

Santesson, R., Symb. Bot. Ups. 12 (1): 349-351. 1952.

Phlyctidium phyllogenum Müll. Arg., Lichenologische Beiträge 28, Flora 71: 141. 1888.

Talo extendido, 4-10 mm diám., generalmente grueso, levemente rugoso, opaco y con cristales, dispuesto superficialmente sobre la epidermis foliar. Apotecios redondeados, primero inmersos en el talo, luego prominentes y en la madurez irregulares y adnatos. Disco hundido, cóncavo, claro, cárneo o amarillento hasta castaño claro no pruinosos, margen prominente con cubierta talina incrustada de cristales. Esporas 9-19 septos transversales, 7$8 \times 34-67 \mu \mathrm{m}$.

Según Lücking (1997) esta especie de Calenia es común en los Neotrópicos. Santesson (1952) la menciona para Honduras, Panamá, Costa Rica y Ecuador.

Material estudiado: ARGENTINA. Misiones, dpto. Iguazú, Parque Nacional Iguazú, sendero Macuco, sobre hojas de Rutaceae, 28-III-95, Ferraro et al. 5012a (CTES); ídem, 4-III-96, Popoff 2894 (CTES); sendero Jacaratia, 8-VIII-95, Maruñak et al. 907 
(CTES); ídem, sobre hojas de Sorocea, 29-III-95, Ferraro et al. 5079 (CTES); ídem, 8-VIII-95, Maruñak et al. 887 (CTES); ídem, Maruñak et al. 904 (CTES, hb. Lücking, hb. Vezda); límite E del Parque Nacional Iguazú, $1200 \mathrm{~m}$ del río Iguazú, sobre frondes de Anemia phylliditis, 11-VIII-1995, Vanni et al. 3514
(CTES); dpto. Guaraní, Predio Guaraní, 26054'-59'S, $54^{\circ} 12^{\prime}-18^{\prime} \mathrm{W}$, sendero CIFOR, en selva, sobre hojas de Actinostemon concolor (Euphorbiaceae), 27-IV-1999, Ferraro, Tressens, Keller, Maruñak y Revilla 6059 (CTES); ídem, sendero a la Aldea Aborigen, en selva, Ferraro et al. 6254 y 6258 (CTES).

\section{Clave para la identificación de las especies}

1. Esporas transversalmente septadas. Talo finamente verrugoso, protalo ausente, hifóforos blancos. Apotecios con margen prominente, sobre todo en apotecios adultos.

2. Apotecios con disco pálido, blanco amarillento.

$2^{\prime}$. Apotecios con disco oscuro, generalmente gris.

Calenia phyllogena (Müll. Arg.) R. Sant.

Calenia depressa Müll. Arg.

$1^{\prime}$. Esporas muriformes. Talo liso, no verrugoso generalmente con abundantes apotecios, hifóforos castaños, no siempre presentes. Apotecios con margen poco desarrollado y disco verdoso, pruinoso.

Calenia monospora Vezda

Tricharia Fée emend. R. Sant.

En el área de estudio se registraban 7 especies, actualmente se conocen 8 , más una variedad.

T. cuneata Ferraro \& Vezda (1989) fue descrita de la provincia de Formosa y últimamente fue coleccionada en la provincia del Chaco (Schinini 25385, CTES) y en Paraguay en el depto. Itapúa (Ferraro 3631, CTES).

\section{Tricharia urceolata var. paraguayensis}

Ferraro \& Lücking var. nov.

Fig. 3, A

A varietate urceolata apotheciis maioribus flavescenter fusca instructis differt.

Holotypus: PARAGUAY. Itapúa, Isla Yacyretá, $56^{\circ} 41^{\prime} \mathrm{W}, 27^{\circ} 24^{\prime} \mathrm{S}$, foliícola, sobre hojas de Trichilia sp. (Meliaceae), Nov. 1988, Ferraro et al. 3668, CTES. Isotypus: hb. Vezda.

Difiere de la variedad urceolata en que los apotecios son más anchos, menos urceolados y con una capa granulosa, castaño-amarillenta sobre el epitecio, similar a una pruina.

Pruina blanquecina ocurre en el epitecio de $T$. carnea, mientras que en $T$. farinosa la pruina es castaño oscura a castaño-chocolate, los apotecios son planos, no urceolados y sin margen visible..
Una sección longitudinal del apotecio, muestra sobre el epitecio una capa granulosa, muy visible debido al color (Fig. 4, A-B). Los ejemplares de T. urceolata analizados hasta el momento no presentan esta característica.

Las esporas de T. urceolata var.paraguayensis son muriformes, alargadas y frecuentemente curvadas (Fig. 4, C).

Consideramos a esle nuevo taxón como variedad ya que las diferencias con $T$. urceolata son, a nuestro criterio, finas y están restringidas geográficamente.

El holotipo muestra, junto a apotecios funcionales, otros evidentemente muy maduros y deformados, de mayor tamaño. Son estructuras semejantes a apotecios que, en realidad, son conidiofóros. Alcanzan un diámetro de 0,5 a $1 \mathrm{~mm}$, el color es castaño, igual al que presentan los apotecios jóvenes, el epitecio se observa más plano; en corte longitudinal se puede ver, que en el interior llevan de 3 a 4 "bolsas de conidios" separadas por una pared de hifas (Fig. 4, D-E). Los apotecios funcionales, productores de ascos y esporas dejan de serlo y se transforman en conidióforos (Vobis, 1980), este mecanismo ha sido a menudo observado en otros grupos de líquenes (Vobis, com. pers.).

El ejemplar Vanni 3506 también presenta conidios en estructuras diferentes de las antes mencionadas. Estos conidióforos son más o menos urceolados, pequeños, negros, se ubi- 

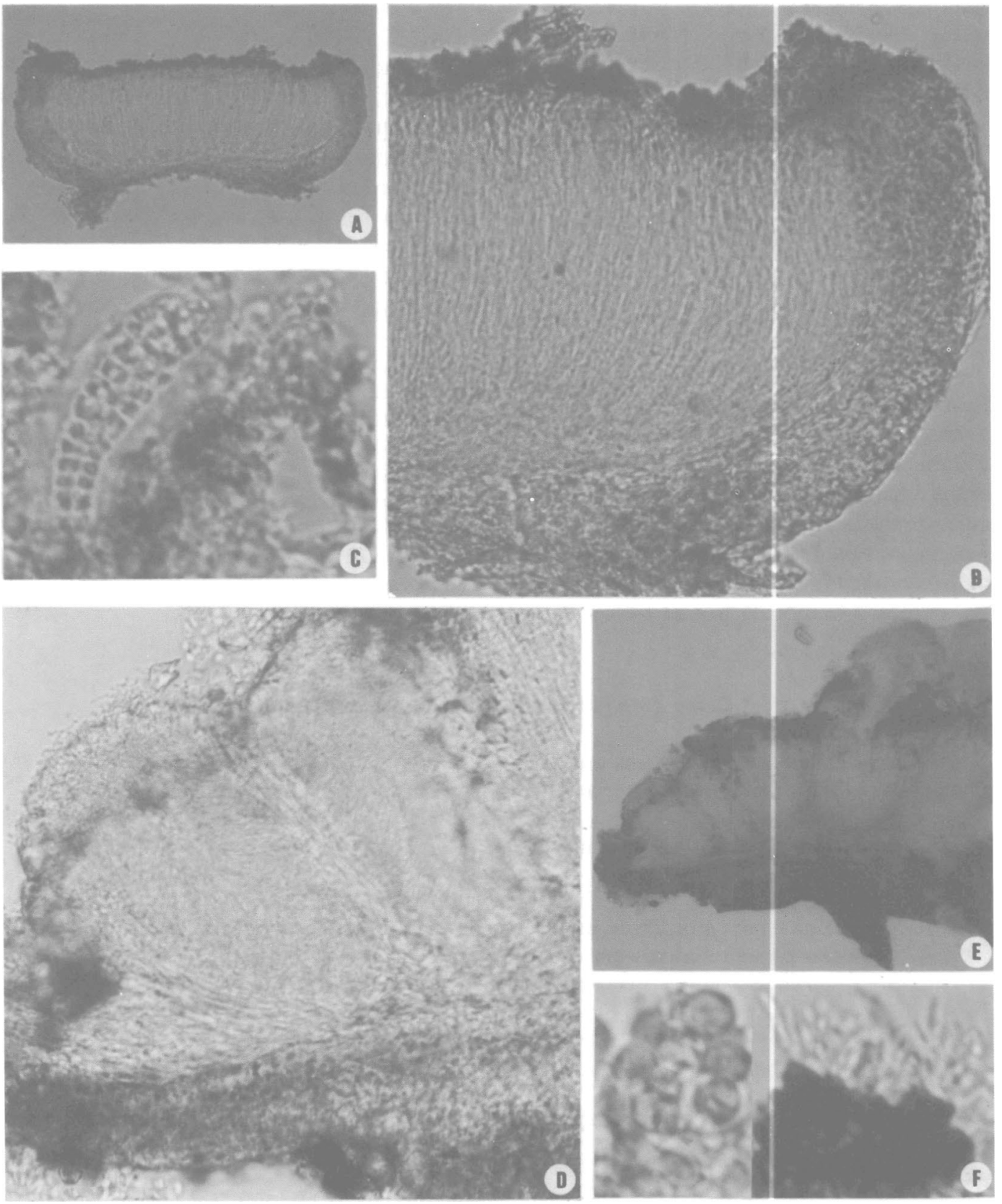

Fig. 3. Tricharia urceolata var. paraguayensis. A-B, corte longitudinal de apotecios. C, espora muriforme. D- E, corte longitudinal de apotecios no funcionales, conidióforos. F, algas y conidióforos del talo de T. urceolata var. paraguayensis (AE: Ferraro 3668, CTES. F: Vanni 3506, CTES). 
can sobre el talo y producen conidios cilíndricos de $3 \times 8 \mu \mathrm{m}$ (Fig. 4, F).

En los ejemplares estudiados no se han encontrado los hifóforos típicos del género.

Material adicional examinado: ARGENTINA. Misiones, dpto. Iguazú, Parque Nacional Iguazú, límite E del Parque Nacional, 1200 m del río Iguazú, 11-VIII-95, Vanni et al. 3506 (CTES). PARAGUAY. Misiones, Isla Yacyretá, foliícola, sobre hojas de Plinia rioularis (Myrtaceae), 25-XI-1989, Ferraro et al. 3626 (CTES).

Tricharia purulhensis Lücking \& Barillas Fig. 3, B

Lücking, R. \& R. Barillas, Bibl. Lichenol. 65: 88. 1997.

Talo inconspicuo, liso, delgado, continuo, gris-verdoso hasta gris-oscuro, provisto de abundantes setas largas, blancas o blanco-amarillentas hasta $1 \mathrm{~mm}$ long., muy llamativas, siempre presentes.

Apotecios hasta 0,5 mm diám., redondeados, disco amarillento, castaño-claro hasta grisáceo, primero cubiertos por el talo y luego emergentes, finalmente prominentes, con el margen cubierto de 5-6 lóbulos, que son el resultado, de los restos de talo que lo cubrían al principio.

Esporas muriformes, oblongas, 1 por asco, $45-69 \times 20-30 \mu \mathrm{m}$.

T. purulhensis y T. albostrigosa son hasta el momento, las únicas especies con pelos o setas blancas que se encuentran en el área de estudio, el resto de las especies presenta pelos negros estériles.

T. purulhensis fue descrita para Costa Rica, por lo que destaco las colecciones realizadas, ya que amplían considerablemente el área de distribución conocida para la especie.

Material estudiado: ARGENTINA. Misiones, dpto. Iguazú, Parque Nacional Iguazú, paseos inferiores, sobre Sorocea, 29-II-1.996, Vanni, Kurtz, Popoff y Herrera 3642 (CTES); dpto. Guaraní, Predio Guaraní, 26 $54^{\prime}-59^{\prime} \mathrm{S}, 54^{\circ} 12^{\prime}-18^{\prime} \mathrm{W}$, sendero CIFOR, en selva, sobre hojas de Pilocarpus, 27-IV-99, Ferraro, Tressens, Keller, Maruñak y Revilla 6044 (CTES, hb. Lücking). Salta, dpto. Orán, Finca San Andrés, en Yungas, selva secundaria, $38^{\circ} 04^{\prime} 23^{\prime \prime} \mathrm{S}, 64^{\circ} 45^{\prime} 07^{\prime \prime} \mathrm{W}$, 28-X-97, Schinini 34177 (CTES).

\section{Clave para la identificación de las especies}

1. Talo con setas blancas. Ascos con una única espora.

2. Apotecio cubierto por 5-6 lóbulos triangulares que corresponden a restos del talo. Talo liso.

$2^{\prime}$. Apotecio sin restos talinos. Talo verrugoso.

T. purulhensis Lücking \& Barillas

$1^{\prime}$. Talo con setas negras.

T. albostrigosa R. Sant.

3. Ascos con una espora muriforme, multicelular, a veces las células se separan originando conidios. 4. Apotecios conspicuamente pruinosos.

5. Pruina clara, blanquecina o grisácea. Apotecios hasta $1 \mathrm{~mm}$ diám.

T. carnea (Müll. Arg.) R. Sant.

5'. Pruina castaña, gruesa, cubriendo el disco y el margen del àpotecio. Apotecios pequeños, hasta $0,4 \mathrm{~mm}$.

T. farinosa R. Sant.

$4^{\prime}$. Apotecios sin pruina, con pruina inconspicua o con una capa epitecial que recuerda a una pruina.

6. Apotecios urceolados.

7. Apotecios pequeños, constrictos en la base, disco hundido con pruina inconspicua blanquecina.

T. urceolata (Müll. Arg.) R. Sant.

$7^{\prime}$. Apotecios grandes, los adultos hasta $1,5 \mathrm{~mm}$ diám., epitecio cubierto por una capa granulosa, castaño-amarillenta que semeja una pruina.

T. urceolata var. paraguayensis Ferraro \& Lücking 
$6^{\prime}$. Apotecios planos, más o menos adheridos al sustrato por toda su superficie.

T. vainioi R. Sant.

3'. Ascos con más de una espora muriforme, generalmente 2-4 esporados.

8. Apotecios oscuros, hifóforos cortos, con los ápices cuneados siempre presentes entre los pelos negros.

8'. Apotecios claros, hifóforos largos, cuneados, angostos.

T. cuneata Ferraro \& Vezda

T. pallida Vezda

\section{Agradecimientos}

Agradezco a los Dres. Antonin Vezda (Brno, República Checa) y Robert Lücking (Bayeruth, Alemania) su estímulo constante y la colaboración prestada para el estudio de los líquenes foliícolas. Al Dr. Orlando Popoff su disposición y ayuda.

Parte de este trabajo fue realizado con el apoyo económico de la Secretaría de Ciencia y Técnica de la Universidad Nacional del Nordeste (Corrientes, Argentina) en el marco del PI 358.

\section{Bibliografía}

CAVALCANTE, W. de.A.,A.A.S.A.S. CAVALCANTE\&F, de B. I.FAI.. 1972. Coletãnea de liquens imperfeitos. Publ. Inst. Micol. Univ. Fed. Pernambuco 647: 1-46.

FERRARO, L.I. 1997. Checklist of foliicolous lichenized fungi from north-eastern Argentina (Corrientes and Misiones), with notes on adjacent areas of Paraguay and Brazil. Abstr. Bot. 21(1): 53-63.

FERRARO, L.I. \& A. VEZDA. 1989. Tricharia cuneata Ferraro \& Vezda sp. nov., Liquen folí́cola del NE de Argentina.
Bonplandia 6(2): 111-115.

KALB, K. \& A. VEZDA. 1988. Neuc oder bemerkenswerte Arten der Flechtenfamilie Gomphillaceae in der Neotropis. Bibl. Lichenol. 29: 1-80, 39 pl.

LÜCKING, R. 1997. Additions and corrections to the knowledge of the foliicolous lichen flora of Costa Rica. The family Gomphillaceae. Bibl. Lichenol. 65: 1-109.

SANTESSON, R. 1952. Foliicolous lichens I. A revision of the taxonomy of the obligately foliicolous, lichenized fungi. Symb. Bot. Ups. 12(1): 1-590.

SÉRUSIAUX, E. \& J.R. DE SLOOVER. 1986. Taxonomical and ecological observations on foliicolous lichens in northern Argentina, with notes on the hyphophores of Asterothyriaceae. Veröff. Geobot. Inst. ETH, Stiftung Rübel, Zürich 91: 260-292.

VEZDA, A. 1973. Foliicole Flechten aus der Republik Guinea (W-Afrika): I. Acta Musei Silesiae, Opava, Ser. A 22: 67-90.

—1979. Flechtensystematische Studien XI. Beiträge zuir Kenntnis der familie Asterothyriaceae (Discolichenes). Folia Geobot. Phytotax. (Praha), 14: 43-94.

VEZDA, A. \& J. POELT. 1987. FlechtensystematischeStudien XII. Die Familie Gomphillaceae und ihre Gliederung. Folia Geobot.. Phytotax. (Praha), 22: 179-198.

VOBIS, G. 1980. Bau und Entwicklung der FlechtenPycnidien und ihrer Conidien. Bibl. Lichenol. 14: 1$141 ; 215$ figs.

WATSON, W. 1929. The classification of lichens. PartI. New Phytol. 28(1): 1-36 